\title{
Le devoir de publier
}

\author{
par Cynthia Jackevicius
}

A lors que je consultais, il y a peu de temps, la liste des lauréats et lauréates des prix de la Société canadienne des pharmaciens d'hôpitaux, j'ai été impressionnée par la quantité de travaux ayant pour sujet l'utilisation appropriée des médicaments! Je me suis dit, "j'espère qu'ils seront publiés ». Peu de temps après, je me suis aperçue que j'acceptais la possibilité qu'une personne ayant passé quelques années sur un projet pourrait le terminer et recevoir un prix, mais, contre toute attente, sans en publier les résultats. Bien que cela puisse sembler étrange, je suis certaine que nous nous sommes tous déjà retrouvés dans une situation similaire. Nous pouvons même croire normal d'omettre parfois la publication du produit de nos recherches. Levez la main ceux et celles qui ont déjà eu un projet de recherche bloqué au stade de résumé beaucoup plus longtemps que nécessaire. $\mathrm{Si}$, comme moi, vous avez levé la main, alors vous êtes un être humain normal qui n'est pas nécessairement doté de superpouvoirs de rédaction et a simplement relégué son manuscrit dans les limbes.

Encore récemment, j'aidais un collègue à rédiger un message à propos de son excellente étude dont le manuscrit aux fins de publication se faisait attendre depuis un peu trop longtemps. Je l'ai encouragé à ne pas abandonner tout juste devant la ligne d'arrivée du marathon de recherche, car je remarquais que l'énergie pour conclure le projet commençait à lui manquer. Mais un projet de recherche ne tombe pas toujours dans les limbes des manuscrits. Parfois, les résultats sont tellement intéressants que l'on veut publier immédiatement! Parfois, lorsqu'une promotion ou l'évaluation du rendement exigent que l'on publie, on est facilement motivé. D'autres fois, la fierté que l'on tire de son travail donne l'énergie pour franchir la ligne d'arrivée. Mais étant donné que près de $50 \%$ des résumés présentés aux rencontres scientifiques n'évoluent jamais jusqu'à la publication des manuscrits, je crois que nous sommes nombreux dans cette situation $^{1}$. Alors, pourquoi les projets tombent-ils dans les limbes des manuscrits et que peut-on faire pour mener nos projets à terme?

Est-ce parce que notre cursus scientifique ne comprend pas de formation en rédaction? C'est possible. Mais il existe tout de même des guides de rédaction scientifique ${ }^{2,3}$. Je sais qu'il m'a fallu du temps pour développer mes compétences en rédaction.
Chemin faisant, j'ai aussi profité de l'enseignement de plusieurs mentors d'écriture. Peut-être que ceux qui ont des aptitudes en rédaction ne réalisent pas l'importance que leurs enseignements auraient pour les néophytes en écriture. Mais ça demande aussi du temps, du tâtonnement et de la persévérance. On dit que, pour apprendre à bien rédiger et constater des progrès sensibles, il faut écrire tous les jours, car c'est en forgeant qu'on devient forgeron.

En ce qui concerne l'écriture, le plus difficile est de commencer selon moi. Comment surmonter cet obstacle? Le nœud du problème se résume à prioriser la rédaction et à s'atteler au travail. Plus facile à dire qu'à faire? Notre horaire est surchargé et l'établissement des priorités peut être difficile. Je trouve très utile les conseils simples mais pleins de sagesse de Stephen Covey dans son livre à succès Les 7 habitudes de ceux qui réalisent tout ce qu'ils entreprennent ${ }^{4}$. Il y propose de modifier légèrement notre façon de voir les choses : «Il faut [...] organiser vos propres priorités au lieu de donner priorité à ce qui figure sur votre emploi du temps. " Covey recommande de répartir nos activités dans quatre cadres selon leur importance et leur urgence, une méthode que je trouve efficace. Pour moi, comme pour beaucoup de gens, ce sont les activités du Cadre I, importantes et urgentes (les crises et les problèmes pressants), dont je viens à bout en premier. Mais les activités du Cadre II, importantes sans être urgentes (comme rédiger des textes sans délai précis), sont souvent celles qui sont mises de côté. En effet, les activités du Cadre III, qui sont (ou semblent être) urgentes sans être vraiment importantes (interruptions, bon nombre de courriels et de réunions), semblent accaparer une large part de notre temps précieux. Pire encore, les activités du Cadre IV, ni urgentes ni importantes (écouter nonchalamment des séries en rafale sur Netflix ou vérifier machinalement les médias sociaux - quelqu'un reconnaît ces occupations " absorbantes »?), sont les vraies chronophages. Lorsqu'il me vient à l'esprit de donner priorité aux activités du Cadre II, surtout au moment de la journée où je suis particulièrement alerte, je suis en mesure de faire avancer les dossiers plus importants, comme la rédaction de manuscrits.

Lorsqu'on a besoin d'encore plus de motivation pour dissiper notre inertie, le court livre humoristique Avalez le 
crapaud! de Brian Tracy offre des idées enrichissantes 5 . Mark Twain a déjà dit que la première chose à faire le matin est d'avaler un crapaud; ainsi, on passe le reste de la journée en sachant que la pire chose est probablement déjà survenue. Selon Tracy, notre " crapaud » est l'activité la plus importante à accomplir tout en étant celle le plus souvent remise au lendemain. Pour nous, ledit " crapaud " est le manuscrit qui coasse à notre oreille et attend qu'on l'écrive.

Mis à part ces approches qui peuvent changer légèrement mais fondamentalement notre perspective, j’ai réfléchi au motif réel qui nous pousse à rédiger des manuscrits. Cela peut paraitre évident, mais le but ultime de nos recherches est d'aider les patients. Et comment cet objectif peut-il nous inciter à la publication de nos recherches? Nombreux sont les pharmaciens qui restent avec plaisir de 10 à 15 minutes de plus au travail pour aider un patient, car nous savons que nous ferons alors quelque chose pour améliorer sa santé. Les besoins du patient sont tangibles et l'on peut s'identifier à lui à cause du contact direct que l'on a avec lui. Dans ces cas, la satisfaction et, en prime, une montée de dopamine récompensent presque immédiatement nos efforts. En revanche, l'assertion indéfinie et fade " aider les patients en publiant nos résultats de recherche " peut s'avérer trop peu concrète pour stimuler la motivation de bien des gens.

Lorsque la publication de nos résultats de recherche n'offre pas de récompense tangible (comme la fierté, la reconnaissance ou une promotion), puiser la force dans l'altruisme qui nous pousse à soigner chacun de nos patients peut nous éviter de tomber dans les limbes des manuscrits. Du moins, si l'on s'entend pour dire que la publication représente un moyen altruiste d'influencer les soins de nombreux patients et d'améliorer leurs résultats thérapeutiques. Certaines personnes peuvent facilement faire appel à cette motivation et ainsi vite retrouver la raison première qui les a incités à entreprendre l'étude. Mais souvent, après quelques années à s'enliser dans tout ce qui entoure l'organisation du projet de recherche, on peut avoir besoin de se rappeler les motivations de départ qui sont axées sur le patient.

Si nous changeons notre perspective et réalisons que nous aiderons même un seul patient - peut-être en visualisant un patient en chair et en os que nous pourrions aider - en publiant le fruit de notre travail, nous pouvons mesurer toute l'importance de ce geste. Ainsi, je crois que ces méthodes nous disposent à accorder quelques minutes supplémentaires ici et là à un projet et à la nécessité de rédiger.

Mis à part l'altruisme qui nous pousse à agir, je me demandais aussi si l'on ne pouvait pas dire qu'il est contraire à l'éthique, pour nous, de retarder la diffusion de nos recherches. Nous sommes souvent critiques envers les compagnies pharmaceutiques qui musellent ou retardent la publication d'essais menés à terme, qualifiant l'acte contraire à l'éthique. Peut-être devrions-nous considérer le fait de retarder la publication de nos propres recherches tout aussi peu éthiques.

Enfin, j’ai présenté quelques idées - des mentors d'écriture, des cadres de gestion du temps, des crapauds, l'altruisme et l'éthique - comme autant d'outils potentiels qui peuvent vous aider à éviter les limbes des manuscrits. Je vous exhorte donc à ne pas voir la publication comme quelque chose de bien à faire un de ces jours, mais plutôt à faire ceci : accorder chaque jour quinze minutes pour prendre à bras le corps vos activités du Cadre II, avaler votre crapaud et revoir votre code d'éthique. Mais avant tout, je vous incite à considérer non seulement que publier nos recherches est un geste altruiste, mais qu'il est aussi de notre devoir de le faire pour améliorer les soins aux patients.

[Traduction par l'éditeur]

\section{Références}

1. Scherer RW, Ugarte-Gil C, Schmucker C, Meerpohl JJ. Authors report lack of time as main reason for unpublished research presented at biomedical conferences: a systematic review. J Clin Epidemiol. 2015;68(7):803-10.

2. Edwards DJ. Dissemination of research results: on the path to practice change. Can J Hosp Pharm. 2015;68(6):465-9.

3. Wagner PD. Writing up your research results for publication. Chest. 2009; 136(2):639-42.

4. Covey SR. Les 7 habitudes de ceux qui réalisent tout ce quils entreprennent. New York (NY): Free Press; 1989.

5. Tracy B. Avalez le crapaud! San Francisco (CA): Berrett-Koehler Publishers, Inc; 2007.

Cynthia Jackevicius, B. Sc. Phm., Pharm. D., M. Sc., est professeure titulaire du Département de pratique pharmaceutique de la Western University of Health Sciences, à Pomona, en Californie, et chercheuse auxiliaire principale de I'Institute for Clinical Evaluative Sciences, à Toronto, en Ontario. Elle est également rédactrice adjointe du Journal canadien de la pharmacie hospitalière.

Intérêts concurrents : Aucun déclaré.

Adresse de correspondance :

Dre Dre Cynthia Jackevicius

College of Pharmacy

Western University of Health Sciences

309 E Second Street

Pomona CA 909469-5527

Courriel : cjackevicius@westernu.edu 